EPJ Web of Conferences 22, 00008 (2012)

DOI: $10.1051 /$ epjconf/20122200008

(C) Owned by the authors, published by EDP Sciences, 2012

\title{
Symmetry considerations in structural phase transitions
}

\author{
J.M. Perez-Mato, M.I. Aroyo and D. Orobengoa \\ Departamento Física de la Materia Condensada, Facultad de Ciencia y Tecnología, \\ Universidad del País Vasco, Apto. 644, 48080 Bilbao, Spain
}

\begin{abstract}
The most important symmetry arguments to be considered in the analysis of structural phase transitions are reviewed. A practical approach is used, with the discussion of many examples. In particular, we stress the straightforward application of computer tools freely available in internet to solve these symmetry-related problems. We focus on programs available on the Bilbao Crystallographic Server (www.cryst.ehu.es), but also the use of some programs from the ISOTROPY site (stokes.byu.edu/isotropy.html) is discussed.
\end{abstract}

\section{INTRODUCTION}

The application of group theory to the investigation of structural phase transitions goes back to the work of Landau (see [1] and references therein) and remains since then a fundamental part of any serious study of solid-solid phase transitions having some kind of symmetry break. If a certain solid phase can be related with some phase of higher symmetry (real or virtual), a systematic use of the group-subgroup relation between the space groups of the two phases is the clue for understanding or predicting its fundamental properties. Essentially, the structural degrees of freedom and the tensor properties can be classified according to irreducible representations (irreps) of the space group of the parent highsymmetry phase, and some particular irrep(s) can be identified as active, in the sense of being the irrep(s) of the degrees of freedom that are responsible for the stabilization of the phase and for the observed symmetry break [2]. This permits to separate in the crystal primary and secondary features, which have rather different thermodynamic behavior and respond differently to external perturbations. In general a consistent use of symmetry arguments can be the key for solving various problems in the research of phase transitions, as for instance: determination of possible symmetries in a phase diagram, ferroic properties, identification of fundamental and marginal degrees of freedom, detection of hidden structural correlations, reduction of the effective number of atomic positional parameters in structural refinements, quantitative comparison of structures with the same or different space group symmetries, systematic characterization of thermal behavior, rationalization of phase diagrams in families of compounds, etc.

The first step in the symmetry characterization of a structural phase transition is to identify the two space groups involved, group-subgroup related, which describe the symmetries of the parent and the low-symmetry phase. Often it is difficult to identify the space group of the low-symmetry phase, while the parent symmetry is wellknown. Sometimes the reverse situation can happen. As we will see below, it is sufficient to possess a minimal experimental information about the lattice change in the transition to be able to limit drastically the possible symmetries of the investigated phase. A second and equally important step is the identification of the active irrep, i.e. the irrep corresponding to the symmetry of the agent that is driving the stabilization of the low-symmetry phase. These two steps are strongly correlated. From the knowledge of the two space groups, the presence of one or several active irreps can be assessed. On the other hand, even without knowing the space group of a phase one can

This is an Open Access article distributed under the terms of the Creative Commons Attribution-Noncommercial License 3.0, which permits unrestricted use, distribution, and reproduction in any noncommercial medium, provided the original work is properly cited. 
have from comparative studies some guess of its active irrep, and from this knowledge the possible symmetries of the low-symmetry phase can be limited to a small set of space groups. A more elaborate but very powerful use of group theory within the field is the description of distorted structures in terms of symmetry-adapted modes. The symmetry-mode decomposition of a low-symmetry phase brings in general a unique insight into the transition mechanism governing the phase stabilization.

In the following we discuss the basic symmetry considerations outlined above, and by means of several examples, we show how computer tools available in internet, in particular some of those present on the Bilbao Crystallographic Server [3,4] at http://www.cryst.ehu.es can help to get adequate and rapid solutions to some of the most basic symmetry problems one can encounter when investigating a structural phase transition. We finish with a brief introduction, by means of a new example, to the possibilities that the recent program AMPLIMODES [5] opens for the symmetry-mode analysis of any displacive distorted structure.

\section{NOT ALL SYMMETRY-BREAKS ARE EQUALLY PROBABLE. ISOTROPY SUBGROUPS}

The Landau theory of symmetry-breaking structural phase transitions (see chapter by Toledano) is based on the basic postulate that the symmetry break taking place in such transitions is due to the condensation (i.e. the change from zero to a non-zero amplitude) of one or a set of collective degrees of freedom that transform according to a single irreducible representation (irrep) of the space group of the high-symmetry phase (the so-called active irrep). These amplitudes $\left\{Q_{i}, i=1, \ldots, n\right\}$ that become spontaneously non-zero in the low-symmetry phase, constitute the so-called order parameter, and the $n$-dimensional irrep describing its transformation properties is usually called the active irrep of the transition $[1,2,6]$.

Although Landau theory may not reproduce accurately the thermodynamic behavior close to the transition points, its symmetry aspects and resulting restrictions are of much general validity. Its basic postulate of a single active irrep is fulfilled in most group-subgroup phase transitions, even if they are discontinuous (for which its validity is not ensured). That the symmetry change in a transition is fully determined by a single active irrep, independently of the condensation of other degrees of freedom, is a very restrictive condition, which can be very powerful in the analysis and prediction of the structural and symmetry properties. In many cases we know the space group of the high-symmetry phase and the active irrep, and we want to know the possible symmetries of the low symmetry phase that the identified order parameter can produce. This reduces to the mathematical problem of determining the so-called isotropy subgroups associated to this specific irrep [7].

Let us call $\mathcal{G}$ the space group of the high-symmetry phase, and $\tau$ the ( $n$-dimensional) active irrep for a certain low-symmetry phase, and let be $\mathbf{Q}=\left(Q_{1}, \ldots, Q_{n}\right)$ the $n$-dimensional order parameter transforming according to the irrep $\tau$. By definition, if an operation $g$ of the space group $\mathcal{G}$ is applied to the low-symmetry distorted structure, the amplitudes $\mathbf{Q}=\left(Q_{1}, \ldots, Q_{n}\right)$ will change/transform according to the irrep $\tau$ :

$$
\boldsymbol{T}_{\tau}(g) \mathbf{Q}=\mathbf{Q}^{\prime}
$$

where the $\boldsymbol{T}_{\tau}(g)$ is the $n \times n$ matrix associated by the irrep $\tau$ to the operation $g$ of $\mathcal{G}$. The system will keep the operation $g$ among its symmetry operations, i.e. $g$ will belong to the low-symmetry space group of the distorted phase, if the system is undistinguishable after the operation is applied, i.e. $\mathbf{Q}^{\prime}=\mathbf{Q}$. Hence, all possible space groups, $\mathcal{H}$ (subgroups of $\mathcal{G}$ ), for the low-symmetry phase, resulting from an active irrep $\tau$, can be obtained from the condition that the space group operations $h$ belonging to $\mathcal{H}$ should fulfill:

$$
\boldsymbol{T}_{\tau}(h) \mathbf{Q}=\mathbf{Q}
$$




\section{Contribution of Symmetries in Condensed Matter}

The possible subgroups of $\mathcal{G}, \mathcal{H}$, that can satisfy this invariance equation, and therefore be the symmetry of a distorted phase with $\tau$ as active irrep, are called isotropy subgroups (for the irrep $\tau$ ) [7].

The lost operations $g$, such that:

$$
\boldsymbol{T}_{\tau}(g) \mathbf{Q}=\mathbf{Q}^{\prime} \neq \mathbf{Q}
$$

transform the structure into configurations which are distinguishable from the original one, but energetically equivalent, and represent domain-related structures [8].

In the case of a 1-dim irrep, eq. (2.2) is rather trivial, and only a single isotropy subgroup exists. It is formed by all operations of $\mathcal{G}$ for which $\boldsymbol{T}_{\tau}(g)=1$. The symmetry of the low-symmetry phase is therefore fully determined by the 1-dim irrep, and very simple to derive.

Let us consider for instance the example of a material having $I 4 / \mathrm{mmm}$ symmetry and consider the possible phase transitions/symmetry breaks that the material can suffer, without changing its unit cell, i.e. without loosing any lattice translation. By definition, the matrix $\boldsymbol{T}_{\tau}(\boldsymbol{E}, \boldsymbol{l})$ associated with a lattice translation $(\boldsymbol{E}, \boldsymbol{l})$ by an irrep $\tau$ is a diagonal matrix of the form $(\boldsymbol{E}$ is here the identity operation):

$$
\boldsymbol{T}_{\tau}(\boldsymbol{E}, \boldsymbol{l})=\left(\begin{array}{lll}
\exp \left(i \mathbf{k}_{1} \cdot \boldsymbol{l}\right) & & \\
& \cdot & \\
& \cdot & \\
& & \cdot \\
& & \exp \left(i \mathbf{k}_{s} \cdot \boldsymbol{l}\right)
\end{array}\right)
$$

where $\left\{\mathbf{k}_{1}, \ldots, \mathbf{k}_{s}\right\}$ are the wave vectors of the star of the irrep [2]. Therefore, according to eq. (2.2), if we want all lattice translations $(\boldsymbol{E}, \boldsymbol{l})$ be maintained in the isotropy subgroup, the wave vectors $\mathbf{k}_{i}$ should be zero, i.e. the active irrep should have null wave vector, or in other words correspond to the point $\Gamma$ of the Brillouin zone.

The irreps of a space group at the Brillouin zone center are equivalent to those of the corresponding point group. The lattice translations have associated identity operators. We can then obtain the irreps of $I 4 / \mathrm{mmm}$ at the $\Gamma$ point by looking at the irreps of the point group $4 / \mathrm{mmm}$ (see Figure 1 ). They can for instance be obtained using POINT on the Bilbao Server [4]. To determine the space groups resulting from each 1-dim irrep acting as active irrep, we have just to keep the operations with character 1 . Table 1 summarizes the result. The irrep $\mathrm{A}_{1 g}$ is trivial and does not break the symmetry. We have taken into account that the columns in Figure 1 correspond to conjugacy classes with several operations. Note that the lattice is labelled as $F$ in the case of the irrep $\mathrm{B}_{2 g}$, despite that the lattice is not changed, because in this case the operations of the orthorhombic group $\mathrm{mmm}$ are defined along the diagonal directions on the plane $x y$ of the tetragonal $I$ lattice. The $I$ lattice described in a unit cell with (110) and (110) as basic translations becomes an $F$ lattice.

The last column of the table in Figure 1, headed with 'functions', contains useful additional information. It lists functions of the coordinates $x, y, z$ of a polar vector, or the coordinates $J_{x}, J_{y}$ and $J_{z}$, of an axial vector, that transform according to the corresponding irrep. For instance, the product $x y$ transforms according to the irrep $\mathrm{B}_{2 g}$. But a shear strain $\epsilon_{x y}$ of the crystal transforms as a function $x y$. Therefore a shear strain $\epsilon_{x y}$ is a crystal magnitude transforming according to $\mathrm{B}_{2 g}$, and could be the order parameter for a phase transition $I 4 / \mathrm{mmm} \longrightarrow F m m m$, which would be then a proper ferroelastic transition, with a switchable spontaneous strain.

In general, irreps of dimension $n>1$ have more than one isotropy subgroup depending on the direction taken by the order parameter $\mathbf{Q}$ within its $n$-dimensional space. In our example, we have two 2-dim irreps, $\mathrm{E}_{g}$ and $\mathrm{E}_{u}$. Let us consider the irrep $\mathrm{E}_{g}$, whose matrices can be chosen in the form indicated in Table 2 (it is straightforward to derive them from the fact that according to the last column of Figure 1 they should correspond to the transformation properties of the pair of functions $x z, y z$ ). As 
Character Table

\begin{tabular}{|c|c|c|c|c|c|c|c|c|c|c|c|c|}
\hline $\mathrm{D}_{4 \mathrm{~h}}(4 / \mathrm{mmm})$ & \# & 1 & 2 & 4 & $2 \mathrm{~h}$ & $2 h^{\prime}$ & -1 & $m_{z}$ & -4 & $m_{v}$ & $m_{d}$ & functions \\
\hline Mult. & - & 1 & 1 & 2 & 2 & 2 & 1 & 1 & 2 & 2 & 2 & . \\
\hline$A_{1 g}$ & $\Gamma_{1}^{+}$ & 1 & 1 & 1 & 1 & 1 & 1 & 1 & 1 & 1 & 1 & $x^{2}+y^{2}, z^{2}$ \\
\hline $\mathrm{A}_{2 \mathrm{~g}}$ & $\mathrm{r}_{2}^{+}$ & 1 & 1 & 1 & -1 & -1 & 1 & 1 & 1 & -1 & -1 & $\mathrm{~J}_{\mathrm{z}}$ \\
\hline $\mathrm{B}_{1 \mathrm{~g}}$ & $\Gamma_{3}^{+}$ & 1 & 1 & -1 & 1 & -1 & 1 & 1 & -1 & 1 & -1 & $x^{2}-y^{2}$ \\
\hline $\mathrm{B}_{2 \mathrm{~g}}$ & $\Gamma_{4}^{+}$ & 1 & 1 & -1 & -1 & 1 & 1 & 1 & -1 & -1 & 1 & $x y$ \\
\hline$E_{g}$ & $\Gamma_{5}^{+}$ & 2 & -2 & 0 & 0 & 0 & 2 & -2 & 0 & 0 & 0 & $(x z, y z),\left(J_{x}, J_{y}\right)$ \\
\hline$A_{1}$ & $\Gamma_{1}^{-}$ & 1 & 1 & 1 & 1 & 1 & -1 & -1 & -1 & -1 & -1 & . \\
\hline $\mathrm{A}_{2 \mathrm{u}}$ & $\Gamma_{2}^{-}$ & 1 & 1 & 1 & -1 & -1 & -1 & -1 & -1 & 1 & 1 & $z$ \\
\hline$B_{1 u}$ & $\Gamma_{3}^{-}$ & 1 & 1 & -1 & 1 & -1 & -1 & -1 & 1 & -1 & 1 & . \\
\hline $\mathrm{B}_{2 \mathrm{u}}$ & $\mathrm{r}_{4}^{-}$ & 1 & 1 & -1 & -1 & 1 & -1 & -1 & 1 & 1 & -1 & . \\
\hline $\mathrm{E}_{\mathrm{u}}$ & $\Gamma_{5}^{-}$ & 2 & -2 & 0 & 0 & 0 & -2 & 2 & 0 & 0 & 0 & $(x, y)$ \\
\hline
\end{tabular}

Figure 1. Character table of the irreps of the point group $4 / \mathrm{mmm}$.

Table 1. Isotropy subgroups of $I 4 / \mathrm{mmm}$ for irreps with $\mathbf{k}=0$. For 2-dimensional irreps only one of the equivalent possible isotropy subgroups is indicated.

\begin{tabular}{c|c|c}
\hline Irrep & Operations conserved & Isotropy Subgroup \\
\hline $\mathrm{A}_{1 g}$ & all operations & $I 4 / m m m$ \\
$\mathrm{~A}_{2 g}$ & $1,2_{z}, 4_{z} \overline{1}, m_{z}, \overline{4}$ & $I 4 / m$ \\
$\mathrm{~B}_{1 g}$ & $1,2_{z}, 2_{h}\left(2_{x}, 2_{y}\right), \overline{1}, m_{z}, m_{v}\left(m_{x}, m_{y}\right)$ & Immm \\
$\mathrm{B}_{2 g}$ & $1,2_{z}, 2_{h}^{\prime}\left(2_{x y}, 2_{x \bar{y}}\right), \overline{1}, m_{z}, m_{d}\left(m_{x y}, m_{x \bar{y}}\right)$ & $F m m m$ \\
$\mathrm{E}_{g}$ & $1,2_{x}, \overline{1}, m_{x}$ & $C 2 / m$ \\
& $1,2_{x y}, \overline{1}, m_{x y}$ & $C 2 / m$ \\
& $1, \overline{1}$ & $P \overline{1}$ \\
$\mathrm{~A}_{1 u}$ & $1,2_{z}, 4_{z}, 2_{h}\left(2_{x}, 2_{y}\right), 2_{h}^{\prime}\left(2_{x y}, 2_{x \bar{y}}\right)$ & $I 422$ \\
$\mathrm{~A}_{2 u}$ & $1,2_{z}, 4_{z}, m_{h}\left(m_{x}, m_{y}\right), m_{d}\left(m_{x y}, m_{x \bar{y}}\right)$ & $I 4 m m$ \\
$\mathrm{~B}_{1 u}$ & $1,2_{z}, 2_{h}\left(2_{x}, 2_{y}\right), \overline{4} m_{d}\left(m_{x y}, m_{x \bar{y}}\right)$ & $I \overline{4} 2 m$ \\
$\mathrm{~B}_{2 u}$ & $1,2_{z}, 2_{h}^{\prime}\left(2_{x y}, 2_{x \bar{y}}\right), \overline{4}, m_{v}\left(m_{x}, m_{y}\right)$ & $I \overline{4} m 2$ \\
$\mathrm{E}_{u}$ & $1,2_{x}, m_{z}, m_{y}$ & $I m m 2$ \\
& $1,2_{x y}, m_{z}, m_{x y}$ & $F m m 2$ \\
& $1, m_{z}$ & $C m$ \\
\hline
\end{tabular}

the inversion $\overline{1}$ has associated the identity matrix, operations $g$ and $\overline{1} \cdot g$ have the same matrix. Identity and inversion will always fulfill eq. (2.2) and operations $2_{z}, m_{z}, 4_{z}^{+}, \overline{4}_{z}^{+}, 4_{z}^{-}$and $\overline{4}_{z}^{-}$will never fulfill it, independently of the direction of $\mathbf{Q}$. The conservation of the other operations, according to eq. (2.2) depends on the direction of $\mathbf{Q}$. For instance if we consider that the $\mathrm{E}_{g}$ order parameter is of the form $(0, Q)$, i.e. takes the direction $(0,1)$ in the irrep space, $2_{x}$ and $m_{x}$ will be conserved in the distorted structure, and the resulting isotropy subgroup is $C 2 / \mathrm{m} 11$.

It is easy to check, considering the matrices of Table 2 that the whole set of possible isotropy subgroups for $\mathrm{E}_{g}$, depending on the order parameter direction, are those listed in Table 3. If expressed in 


\section{Contribution of Symmetries in Condensed Matter}

Table 2. Matrices of the irrep $\mathrm{E}_{g}$ of the point group 4/ $\mathrm{mmm}$ for a certain choice of the basis in the irrep space.

\begin{tabular}{l||c|c|c|c|c|c|c|c}
\hline & $1, \overline{1}$ & $2_{z}, m_{z}$ & $4_{z}^{+}, \overline{4}_{z}^{+}$ & $4_{z}^{-}, \overline{4}_{z}^{-}$ & $2_{x}, m_{x}$ & $2_{y}, m_{y}$ & $2_{x y}, m_{x y}$ & $2_{x \bar{y}}, m_{x \bar{y}}$ \\
\hline $\mathrm{E}_{g}$ & $\left(\begin{array}{ll}1 & 0 \\
0 & 1\end{array}\right)$ & $\left(\begin{array}{rr}-1 & 0 \\
0 & -1\end{array}\right)$ & $\left(\begin{array}{rr}0 & -1 \\
1 & 0\end{array}\right)$ & $\left(\begin{array}{rr}0 & 1 \\
-1 & 0\end{array}\right)$ & $\left(\begin{array}{rr}-1 & 0 \\
0 & 1\end{array}\right)$ & $\left(\begin{array}{rr}1 & 0 \\
0 & -1\end{array}\right)$ & $\left(\begin{array}{rr}0 & -1 \\
-1 & 0\end{array}\right)$ & $\left(\begin{array}{ll}0 & 1 \\
1 & 0\end{array}\right)$ \\
\hline
\end{tabular}

Table 3. Isotropy subgroups of the irrep $\mathrm{E}_{g}$ of $I 4 / \mathrm{mmm}$, depending on the direction of the order parameter within the irrep space. For each subgroup its conventional basis is indicated in terms of the basis $(\mathbf{a}, \mathbf{b}, \mathbf{c})$ of $I 4 / \mathrm{mmm}$. For completeness the null origin shift between the two unit cells is also listed. The conventional setting with the monoclinic unique axis along the $b$-direction is used.

\begin{tabular}{c|c|c}
\hline Direction & Operations & Space group \\
\hline$(0,1)$ & $1,2_{x}, \overline{1}, m_{x}$ & $C 2 / m(-\mathbf{b}-\mathbf{c}, \mathbf{a}, \mathbf{c} ; 000)$ \\
$(1,0)$ & $1,2_{y}, \overline{1}, m_{y}$ & $C 2 / m(-\mathbf{a}-\mathbf{c}, \mathbf{b}, \mathbf{c} ; 000)$ \\
$(1,1)$ & $1,2_{x y}, \overline{1}, m_{x y}$ & $C 2 / m(\mathbf{a}+\mathbf{b}+\mathbf{c}, \mathbf{a}+\mathbf{b}, \mathbf{c} ; 000)$ \\
$(-1,1)$ & $1,2_{x \bar{y}}, \overline{1}, m_{x \bar{y}}$ & $C 2 / m(\mathbf{a}+\mathbf{b}+\mathbf{c},-\mathbf{a}+\mathbf{b}, \mathbf{c} ; 000)$ \\
arbitrary & $1, \overline{1}$ & $P \overline{1}\left(\mathbf{a}, \mathbf{b}, \frac{1}{2}(\mathbf{a}+\mathbf{b}+\mathbf{c}) ; 000\right)$ \\
\hline
\end{tabular}

a conventional setting, the $I$ centred unit cell must be transformed to a $C$ centred cell for the monoclinic space groups and to a primitive unit cell for the triclinic one. The lattice however does not change, it is only expressed in a conventional unit cell consistent with the direction of the monoclinic axis.

The directions $(0,1)$ and $(1,0)$ for the order parameter are equivalent: they correspond to domain related directions. The four equivalent order parameter values obtained by applying the matrices of the lost operations are $\{(Q, 0),(0, Q),(-Q, 0),(0,-Q)\}$. In mathematical terms, this means that the isotropy subgroups corresponding to the order parameter directions $(0,1)$ and $(1,0)$ belong to the same conjugacy class. In general the number of domain-related configurations is equal to the index of the subgroup (4 in this case). Similarly for the solutions of type $(1,1)$, there are four equivalent order parameter directions $\{(a, a),(-a, a),(-a,-a),(a,-a)\}$.

We have then three possible non-equivalent isotropy subgroups for the irrep $\mathrm{E}_{g}$, which are not equivalent: $C 2 / m$ (with monoclinic axis along $x$ or $y$ ), $C 2 / m$ (with monoclinic axis along the oblique directions $(110)$ or $(1 \overline{1} 0)$ ) and $P \overline{1}$. With a similar analysis for $\mathrm{E}_{u}$ we can complete the set of nonequivalent isotropy subgroups of $I 4 / \mathrm{mmm}$ for irreps with $\mathbf{k}=0$ (see Table 1 ). We have therefore only 13 possible symmetries for the low-symmetry structure that maintain the crystal lattice and comply with the Landau postulate. It is interesting to compare this set of isotropy subgroups with the set of all possible subgroups of $I 4 / \mathrm{mmm}$ that maintain the lattice. These latter can be readily obtained with the program CELLSUB of the Bilbao Crystallographic Server [3].

\subsection{The program CELLSUB}

This program lists all possible subgroups of a given space group for a given $k$-index (or $k$-indices smaller than a certain value). The program lists first the space group types, and then, for each space group type, it can distinguish the different conjugacy classes. The $k$-index $i_{k}$ is the 'klassengleiche' index, and indicates the multiplication factor relating the volume of the primitive cell of the subgroup with respect to the primitive cell of the original structure. Thus, $i_{k}=1$ implies conservation of the primitive unit cell, and therefore of the whole lattice.

The list of subgroups of $I 4 / \mathrm{mmm}$ with $i_{k}=1$ as given by CELLSUB is reproduced in Figure 2. But this first ouput of the program only lists the possible space group types appearing as subgroups of $I 4 / \mathrm{mmm}$. In order to enumerate all possible non-equivalent subgroups, one has to click over each subgroup type. The program then yields the different conjugacy classes of subgroups for this group type, 
EPJ Web of Conferences

\section{N. of subgroups (for k-index 1 ) found: 18}

\section{t-subgroups of space group $14 / \mathrm{mmm}$ (139)}

\begin{tabular}{|c|c|c|c|c|c|c|}
\hline $\mathbf{N}$ & HM Symbol & ITA & index & t-index & k-index & More info \\
\hline 1 & $I-42 m$ & 121 & 2 & 2 & 1 & show... \\
\hline 2 & $1-4 m 2$ & 119 & 2 & 2 & 1 & show... \\
\hline 3 & $14 \mathrm{~mm}$ & 107 & 2 & 2 & 1 & show... \\
\hline 4 & 1422 & 097 & 2 & 2 & 1 & show... \\
\hline 5 & $14 / m$ & 087 & 2 & 2 & 1 & show... \\
\hline 6 & $1-4$ & 082 & 4 & 4 & 1 & show... \\
\hline 7 & 14 & 079 & 4 & 4 & 1 & show... \\
\hline 8 & Immm & 071 & 2 & 2 & 1 & show... \\
\hline 9 & Fmmm & 069 & 2 & 2 & 1 & show... \\
\hline 10 & Imm2 & 044 & 4 & 4 & 1 & show... \\
\hline 11 & $F m m 2$ & 042 & 4 & 4 & 1 & show... \\
\hline 12 & 1222 & 023 & 4 & 4 & 1 & show... \\
\hline 13 & $F 222$ & 022 & 4 & 4 & 1 & show... \\
\hline 14 & $\mathrm{C} 2 / \mathrm{m}$ & 012 & 4 & 4 & 1 & show... \\
\hline 15 & $\mathrm{Cm}$ & 008 & 8 & 8 & 1 & show... \\
\hline 16 & C2 & 005 & 8 & 8 & 1 & show... \\
\hline 17 & $P-1$ & 002 & 8 & 8 & 1 & show... \\
\hline 18 & $P 1$ & 001 & 16 & 16 & 1 & show... \\
\hline
\end{tabular}

Figure 2. Subgroup types of $I 4 / \mathrm{mmm}$ with $i_{k}=1$, as given by CELLSUB.

i.e. the set of non-equivalent subgroups belonging to this space group type. Figure 3 is a screenshot of this second output for subgroups of type $C 2 / \mathrm{m}$.

According to Figure 3, there are 3 conjugacy classes of space groups of type $C 2 / \mathrm{m}$. Classes 2 and 3 in the list correspond to the two non-equivalent isotropy subgroups discussed above as isotropy subgroups. On the other hand, class 1 has the monoclinic axis along the $z$ direction of the tetragonal setting, and it is therefore not an isotropy subgroup (Note that the ordering and numbering of the classes done by the program is not fixed. Different program runs may order the classes differently).

Inspecting the classes for all subgroup types, one can assess that the number of non-equivalent subgroups with $i_{k}=1$ is 26 , while the number of non-equivalent isotropy subgroups listed in Table 1 is only 13 . Therefore, not all subgroups of $I 4 / \mathrm{mmm}$ compatible with its lattice can be reached through a phase transition with a single active irrep. The Landau postulate restricts considerably the number of possible distinct symmetries.

The restriction of the possible symmetry-break to only isotropy subgroups is in general very demanding, and can provide very valuable information. For instance, if a symmetry change between two phases does not comply with this condition, it indicates that the distorted phase contains at least two active irreps, i.e. the symmetry break can only be explained considering at least two different order parameters transforming according to two different irreps. It will therefore be highly probable that a sequence of at least two thermal phase transitions exist as temperature is increased, due to the separate 
Class 1

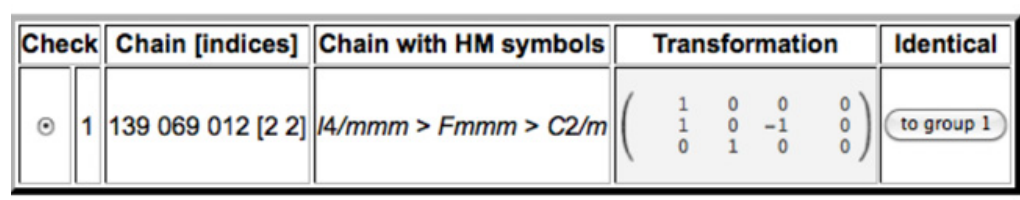

Show graph

Class 2

\begin{tabular}{|c|c|c|c|c|c|c|c|c|}
\hline \multicolumn{2}{|c|}{ Check } & \multirow{2}{*}{$\begin{array}{l}\text { Chain [indices] } \\
139071012 \text { [2 2] }\end{array}$} & \multirow{2}{*}{$\begin{array}{l}\text { Chain with HM symbols } \\
14 / \mathrm{mmm}>\mathrm{Immm}>\mathrm{C} 2 / \mathrm{m}\end{array}$} & \multicolumn{4}{|c|}{ Transformation } & \multirow{2}{*}{$\begin{array}{c}\text { Identica } \\
- \\
-\end{array}$} \\
\hline$\odot$ & 2 & & & $\begin{array}{r}-1 \\
0 \\
-1\end{array}$ & $\begin{array}{l}0 \\
1 \\
0\end{array}$ & $\begin{array}{l}1 \\
0 \\
0\end{array}$ & $\left.\begin{array}{l}0 \\
0 \\
0\end{array}\right)$ & \\
\hline 0 & 3 & 139071012 [2 2] & $14 / \mathrm{mmm}>1 \mathrm{mmm}>\mathrm{C} 2 / \mathrm{m}$ & $\begin{array}{r}0 \\
-1 \\
1\end{array}$ & $\begin{array}{l}1 \\
0 \\
0\end{array}$ & $\begin{array}{l}0 \\
1 \\
0\end{array}$ & $\left.\begin{array}{l}0 \\
0 \\
0\end{array}\right)$ & - \\
\hline
\end{tabular}

Show graph

Class 3

\begin{tabular}{|c|c|c|c|c|c|c|c|c|}
\hline \multicolumn{2}{|c|}{ Check } & \multirow{2}{*}{\begin{tabular}{|l|} 
Chain [indices] \\
139069012 [2 2]
\end{tabular}} & \multirow{2}{*}{\begin{tabular}{|l|} 
Chain with HM symbols \\
$14 / \mathrm{mmm}>\mathrm{Fmmm}>\mathrm{C} 2 / \mathrm{m}$
\end{tabular}} & \multicolumn{4}{|c|}{ Transformation } & \multirow{2}{*}{$\begin{array}{c}\text { Identical } \\
--\end{array}$} \\
\hline$\odot$ & 4 & & & $\begin{array}{r}1 \\
-1 \\
0\end{array}$ & $\begin{array}{l}1 \\
1 \\
0\end{array}$ & $\begin{array}{r}-1 / 2 \\
1 / 2 \\
1 / 2\end{array}$ & $\left.\begin{array}{l}0 \\
0 \\
0\end{array}\right)$ & \\
\hline 0 & 5 & 139069012 [2 2] & $14 / \mathrm{mmm}>\mathrm{Fmmm}>\mathrm{C} 2 / \mathrm{m}$ & $\begin{array}{r}-1 \\
-1 \\
0\end{array}$ & $\begin{array}{r}1 \\
-1 \\
0\end{array}$ & $\begin{array}{l}1 / 2 \\
1 / 2 \\
1 / 2\end{array}$ & $\left.\begin{array}{l}0 \\
0 \\
0\end{array}\right)$ & -- \\
\hline
\end{tabular}

Show graph

Figure 3. Output of CELLSUB indicating the different conjugacy classes of subgroups of $I 4 / \mathrm{mmm}$ of type $C 2 / \mathrm{m}$ with index $i_{k}=1$.

condensation of the two order parameters, and therefore an intermediate phase is most likely (see below for an example).

Some predictive power about the phase diagram of a compound or that of similar compounds can also be achieved from the knowledge of the isotropy subgroups of a certain irrep. If a multidimensional irrep is known to be active in a certain material, it may exhibit several phases with different symmetries corresponding to different isotropy subgroups of this unique active irrep, depending on the thermodynamic stabilization of the order parameter along different directions of the representation space. Similarly, if the same irrep is active in a family of isomorphous compounds, the distorted symmetries observed in different compounds may be different, but will correspond to different isotropy subgroups of the same irrep. 
Table 4. Isotropy subgroups of $P m \overline{3} m$ for irrep $\mathrm{R}_{4}^{+}$. In this table, the triad in the third column indicates for each space group the restricted direction (one of a set of equivalent ones) of the order parameter in its 3-dim space, in the representation basis used in [11].

\begin{tabular}{|c|c|c|c|}
\hline Isotropy subgroups & Basis & Direction & Compound \\
\hline$I 4 / \mathrm{mcm}$ & $(\mathbf{a}+\mathbf{b},-\mathbf{a}+\mathbf{b}, 2 \mathbf{c} ; 000)$ & $(a, 0,0)$ & $\mathrm{SrZrO}_{3}$ \\
\hline Imma & $(\mathbf{a}+\mathbf{c}, 2 \mathbf{b},-\mathbf{a}+\mathbf{c} ; 000)$ & $(a, a, 0)$ & $\mathrm{SrZrO}_{3}$ \\
\hline$R \overline{3} c$ & $-\mathbf{a}+\mathbf{b},-\mathbf{b}+\mathbf{c}, 2 \mathbf{a}+2 \mathbf{b}+2 \mathbf{c} ;\left(\begin{array}{lll}0 & 0 & 0\end{array}\right)$ & $(a, a, a)$ & $\mathrm{CeAlO}_{3}$ \\
\hline$C 2 / m$ & $\left(-2 \mathbf{c}, 2 \mathbf{b}, \mathbf{a}+\mathbf{c} ; 0 \frac{1}{2} \frac{1}{2}\right)$ & $(a, b, 0)$ & $\mathrm{BaPbO}_{3}$ \\
\hline $\begin{array}{c}C 2 / c \\
P \overline{1}\end{array}$ & $\begin{array}{c}\left(-\mathbf{a}+2 \mathbf{b}-\mathbf{c},-\mathbf{a}+\mathbf{c}, \mathbf{a}+\mathbf{c} ; 0 \frac{1}{2} \frac{1}{2}\right) \\
\mathbf{b}+\mathbf{c}, \mathbf{a}+\mathbf{c}, \mathbf{a}+\mathbf{b} ;\left(\begin{array}{lll}0 & 0 & 0\end{array}\right)\end{array}$ & $\begin{array}{l}(a, a, b) \\
(a, b, c)\end{array}$ & $\mathrm{LaCoO}_{3}$ \\
\hline
\end{tabular}

Let us consider for instance the example of the perovskites. Perovskites are known to have often an intrinsic instability due to the softness of three degenerate rigid unit modes (modes tilting the octahedral framework and keeping the $\mathrm{BX}_{6}$ octahedra approximately rigid), which transform according to the 3-dim irrep $\mathrm{R}^{+}{ }^{+}$of the parent symmetry $P m \overline{3} m$ [9]. The isotropy subgroups of $P m \overline{3} m$ for irrep $\mathrm{R}^{+}$ are indicated in Table 4. For each of the possible isotropy subgroups, we have listed one perovskite compound that is known to have this symmetry in one particular phase. One can see that any of the possible symmetries is realized in some perovskite compound, except the lowest one, which corresponds to an arbitrary direction of the order parameter.

There are perovskites, as $\mathrm{CeAlO}_{3}$, where the following phase transition sequence takes place as temperature is increased [10]:

$$
I 4 / m c m \longrightarrow I m m a \longrightarrow R \overline{3} c \longrightarrow P m \overline{3} m
$$

Thus, the system is changing the direction of its $\mathrm{R}_{4}^{+}$order parameter from one phase to the next, through phase transitions, yielding symmetries given by different isotropy subgroups listed in Table 4.

The determination by hand of the isotropy subgroups for a given irrep is time consuming and tedious. Fortunately, the isotropy subgroups of the 230 space groups are listed for irreps corresponding to special points of the Brillouin zone in [7], or they can be obtained automatically using the program ISOTROPY [11], or some other programs, also available in (http://stokes.byu.edu/isotropy.html). The use of ISOTROPY requires getting familiar with some specific program commands. A more direct form to get the information about possible isotropy subgroups for a given irrep is to run the associated programs INVARIANTS or ISODISTORT [12], which are more user-friendly, and can be executed by filling some self-evident menus. These two programs list the isotropy subgroups of a chosen irrep as a preliminary result. The information is more complete in ISODISTORT, but running this program, requires the introduction of some structure for the high-symmetry phase (one can introduce a false one, since a specific structure is not required for deriving the list of isotropy subgroups).

\section{MORE ON THE POSSIBLE SYMMETRY OF A DISTORTED PHASE}

In many practical cases, we do not know the active irrep of the transition, but we may know some specific conditions on the symmetry of the distorted phase coming from experimental observation. These conditions can be sufficient for deriving a limited set of possible space groups $\mathcal{H}$, and possible active irreps.

Let us consider as an example a real case. Crystals that include both fullerene and cubane molecules have been recently synthetized. They are known to crystallize at high temperatures in the $F m \overline{3} m$ space group with the disordered fullerenes centred at the site $4 a(000)$ and disordered cubane molecules at $4 b\left(\frac{1}{2} \frac{1}{2} \frac{1}{2}\right)$ [13]. At low temperature the system exhibits a couple of phase transitions, as the molecules become ordered. From powder diffraction experiments, the symmetry of the final phase has been reported to be a primitive orthorhombic structure, with its lattice parameters satisfying the approximate 


\begin{tabular}{|l|l|l|l|l|l|l|}
\hline N & HM Symbol & ITA & index & t-index & k-index & More info \\
\hline 1 & Pnma & 062 & 24 & 6 & 4 & show... \\
\hline 2 & Pbca & 061 & 24 & 6 & 4 & show... \\
\hline 3 Pbcn & 060 & 24 & 6 & 4 & show... \\
\hline 4 & Pmmn & 059 & 24 & 6 & 4 & show... \\
\hline 5 & Pnnm & 058 & 24 & 6 & 4 & show... \\
\hline 6 & Pbcm & 057 & 24 & 6 & 4 & show... \\
\hline 7 Pccn & 056 & 24 & 6 & 4 & show... \\
\hline 8 & Pbam & 055 & 24 & 6 & 4 & show... \\
\hline 9 & Pcca & 054 & 24 & 6 & 4 & show... \\
\hline 10 & Pmna & 053 & 24 & 6 & 4 & show... \\
\hline 11 & Pnna & 052 & 24 & 6 & 4 & show... \\
\hline 12 & Pmma & 051 & 24 & 6 & 4 & show... \\
\hline 13 & Pban & 050 & 24 & 6 & 4 & show... \\
\hline 14 & Pccm & 049 & 24 & 6 & 4 & show... \\
\hline 15 & Pnnn & 048 & 24 & 6 & 4 & show... \\
\hline 16 & Pmmm & 047 & 24 & 6 & 4 & show... \\
\hline
\end{tabular}

Figure 4. Subgroups (types) of $F m \overline{3} m$ with point group $m m m, i_{k}=4$ and no centring, as given by CELLSUB.

relations $a$ and $b \approx a_{c} / \sqrt{2}$, while $c \approx 2 a_{c}$ ( $a_{c}$ the cell parameter of the cubic phase), but its space group, and therefore its structure could not be determined [14]. Obviously, if we can restrict the symmetry to a minimal set of possible/probable space groups, we could have a better chance for succeeding in the interpretation and analysis of the diffraction diagram of this phase.

We can start by determining all possible primitive orthorhombic subgroups of $F m \overline{3} m$, fulfilling the unit cell relation that has been observed. We can again use for that the program CELLSUB provided that we know the $k$-index of the subgroup we are searching. This index can be derived in a straightforward manner comparing the volumes of the primitive unit cells of the two space groups. For $F m \overline{3} m$, it is $a_{c}^{3} / 4$, while in the orthorhombic phase is $a_{c}^{3}$. This means, the orthorhombic space group keeps only $\frac{1}{4}$ of the lattice translations, or in other words the primitive orthorhombic unit cell contains 4 formula units, while the $F m \overline{3} m$ structure only one. Hence $i_{k}=4$. The point group of the searched orthorhombic space groups can be 222, $\mathrm{mm} 2$ or $\mathrm{mmm}$. To simplify the example, we are going to assume that the point group symmetry is maximal, i.e. $\mathrm{mmm}$ (but we could proceed similarly with the other two possible point groups).

Restricted to $i_{k}=4$, point group $m m m$, and no centring, CELLSUB lists a quite long list of subgroups of $F m \overline{3} m$ that is reproduced in Figure 4. But not all of them will have unit cell parameters consistent with the experimental values. By clicking on 'show' we get the different non-equivalent classes of subgroups of this type and the transformation matrix relating its conventional basis with the one of the cubic group. For instance, for $\mathrm{Pbca}$, there is a single class. Its transformation matrix is $(-\mathbf{b},-\mathbf{c}, \mathbf{a} ; 000)$ or equivalent ones. Hence, its cell parameters coincide with those of the cubic cell. Therefore this space group can be discarded. On the other hand, if we consider subgroups of type Pnma, there are four classes of Pnma subgroups, with one of the classes having as transformation $\left(2 \mathbf{b}, \frac{1}{2}(\mathbf{a}+\mathbf{c}), \frac{1}{2}(\mathbf{a}-\mathbf{c}) ; \frac{1}{4} \frac{3}{4} \frac{1}{2}\right)$ or equivalent. The parameters of the Pnma orthorhombic cell for this class are therefore $a=2 a_{c}, b=c=a_{c} / \sqrt{2}$, which are consistent with the orthorhombic cell observed, if a permutation of the cell parameters labels is done. 
Table 5. Possible centrosymmetric orthorhombic subgroups of $F m \overline{3} m$ fulfilling the lattice metrics observed in the orthorhombic phase of fullerene-cubane crystals. The third column indicates the resulting Wyckoff splitting for the two sites occupied by the centres of the fullerene and cubane molecules. The fourth column shows the corresponding active irrep of the order parameter, in the case that the subgroup is an isotropy subgroup. The third and fourth columns are results obtained with SYMMODES.

\begin{tabular}{c|c|c|c}
\hline subgroup & Basis & $\begin{array}{c}\text { Splitting of } \\
4 a, 4 b \text { orbits }\end{array}$ & Active irrep \\
\hline$P n m a$ & $2 \mathbf{a}, \frac{1}{2}(\mathbf{b}+\mathbf{c}), \frac{1}{2}(-\mathbf{b}+\mathbf{c}) ; \frac{3}{4} \frac{1}{4} 0$ & $4 c, 4 c$ & $\left(0, \frac{1}{2}, 0\right) \mathrm{DT} 5$ \\
$P m m n$ & $\frac{1}{2}(\mathbf{a}-\mathbf{b}), \frac{1}{2}(\mathbf{a}+\mathbf{b}), 2 \mathbf{c} ; \frac{1}{4} 0 \frac{3}{4}$ & $2 a+2 a, 2 a+2 a$ & - \\
$P c c n$ & $\frac{1}{2}(\mathbf{a}-\mathbf{b}), \frac{1}{2}(\mathbf{a}+\mathbf{b}), 2 \mathbf{c} ; \frac{1}{4} 0 \frac{3}{4}$ & $4 c, 4 c$ & - \\
$P m m a$ & $2 \mathbf{a}, \frac{1}{2}(\mathbf{b}+\mathbf{c}), \frac{1}{2}(-\mathbf{b}+\mathbf{c}) ; \frac{1}{2} 00$ & $2 a+2 f, 2 e+2 d$ & $\left(0, \frac{1}{2}, 0\right) \mathrm{DT} 5$ \\
$P c c m$ & $\frac{1}{2}(\mathbf{a}-\mathbf{b}), \frac{1}{2}(\mathbf{a}+\mathbf{b}), 2 \mathbf{c} ; 000$ & $2 e+2 b, 2 a+2 h$ & - \\
$P m m m$ & $\frac{1}{2}(\mathbf{a}-\mathbf{b}), \frac{1}{2}(\mathbf{a}+\mathbf{b}), 2 \mathbf{c} ; 000$ & $2 q+1 f+1 h, 1 a+2 t+1 c$ & - \\
\hline
\end{tabular}

Inspecting the classes for all the subgroups in the list, it is straightforward to conclude that only six symmetries, namely Pnma, Pmmn, Pccn, Pmma, Pccm and Pmmm, with transformations matrices listed in Table 5, are consistent with the experimental observation. It is interesting to compare this table with the analysis done in [17]. The method employed in that work, rather indirect, overlooked the Pnma and Pmmn subgroups, and only four of the six possible space groups were derived.

The list in Table 5 could be further reduced if we assumed that the searched symmetry should correspond to an isotropy subgroup, i.e. a single active irrep must explain the corresponding symmetry break. But this leads into a new type of problem, which we treat in more detail in the following section.

\section{THE INVERSE LANDAU PROBLEM. SECONDARY SPONTANEOUS VARIABLES}

The most important information required for the characterization of a group-subgroup phase transition is the identification of its active irrep. This is the so-called inverse Landau problem. In most cases the knowledge of the high and low space groups is sufficient to identify the active irrep. There are however cases where several different irreps can yield the same symmetry break, and in this case only a detailed decomposition of the structural change into irrep components can elucidate which is the prevailing symmetry component, and determine in this way the active irrep. In any of the two cases, the first step is to derive the possible irreps that have the observed space group of the low-symmetry phase as an isotropy subgroup. The website ISOTROPY (stokes.byu.edu/isotropy.html) includes the program COPL [15], which does this identification, and also provides the irreps of all additional spontaneous secondary variables. In the Bilbao Crystallographic Server, the program SYMMODES, which in fact internally uses an adapted version of COPL (kindly provided by H. T. Stokes), can also give this information as a by-product.

In the low symmetry phase, apart from the order parameter and quantities transforming according to the active irrep, other variables or degrees of freedom transforming according to other irreps can also condense or become spontaneous. The only requirement is that they are compatible with the low-symmetry space group. This is the realization of the Von Neumann principle. In general, any variable/degree of freedom compatible with the symmetry of the crystal is allowed and will therefore in general have a non-zero value. Using the concept of isotropy subgroup, we can say that any quantity with transformation properties given by an irrep having an isotropy subgroup different from $\mathcal{G}$ and containing the group $\mathcal{H}$ of the distorted phase will be spontaneous in the transition (i.e. it will change from zero to non-zero values in the distorted phase). Therefore, while the active irrep must have $\mathcal{H}$ as an isotropy subgroup, the irreps associated to spontaneous secondary variables have in general an isotropy subgroup 
Table 6. Irreps compatible with the symmetry break $I 4 / \mathrm{mmm} \longrightarrow C 2 / m(-\mathbf{b}-\mathbf{c}, \mathbf{a}, \mathbf{c} ; 000)$ obtained with the program COPL [15].

\begin{tabular}{cccc}
\hline Irrep & Dir & Isotropy Subgroup & Size \\
\hline GM1+ & (a) & $139 \mathrm{I} 4 / \mathrm{mmm}$ & 1 \\
GM2+ & (a) & $71 \mathrm{Immm}$ & 1 \\
GM5+ & $(\mathrm{a}, 0)$ & $12 \mathrm{C} 2 / \mathrm{m}$ & 1 \\
\hline
\end{tabular}

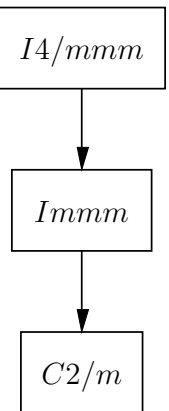

Figure 5. Graph of maximal subgroups connecting the space group $I 4 / \mathrm{mmm}$ and its subgroup $C 2 / \mathrm{m}(-\mathbf{b}-\mathbf{c}$, a, c;000) (or equivalent), obtained with SUBGROUPGRAPH (to obtain this graph one has to choose the correct conjugacy class among the three classes given by the program).

which is a supergroup of $\mathcal{H}$. Let us consider the following simple case:

$$
I 4 / m m m \longrightarrow C 2 / m(-\mathbf{b}-\mathbf{c}, \mathbf{a}, \mathbf{c} ; 000)
$$

From the example discussed above, we know that the active irrep for this symmetry change is $\mathrm{E}_{g}$ $(\mathbf{k}=0)$. Table 6 shows the output of COPL (stokes.byu.edu/isotropy.html) for this symmetry change. The program lists a set of irreps, a 'direction' or subspace within the irrep space and their isotropy subgroup. The final column headed 'size' indicates the multiplication of the primitive unit cell of the corresponding isotropy subgroup relative to the parent high-symmetry space group (i.e. the klassengleiche index $i_{k}$ ). We have then the irrep GM5+ as the active irrep with the observed symmetry as isotropy subgroup, and then a secondary irrep GM2+ with an isotropy subgroup $I \mathrm{mmm}$, which necessarily must be a supergroup of $C 2 / m$. In addition, the trivial irrep GM1+ always appears as possible symmetry of secondary variables that are allowed to have non-zero values both at the distorted phase, and also at the parent phase. The label GM is being used to indicate that they are irreps at the Brillouin zone centre (Gamma point), which do not break the translational symmetry. GM5+ must be the irrep labelled $\mathrm{E}_{g}$ while GM2+ should be the irrep labelled as $\mathrm{B}_{1 g}, c f$. Section $2^{1}$.

The reason for the existence of secondary spontaneous quantities transforming according to the irrep $\mathrm{B}_{1 g}$ becomes obvious if we use the programs SUBGROUPGRAPH $[3,16]$ or CELLSUB to construct the graph of maximal subgroups connecting the two end space groups. This is shown in Figure 5. The group $I m m m$, which is the isotropy subgroup of $\mathrm{B}_{1 g}$, is indeed a supergroup of $C 2 / \mathrm{m}$. Therefore, variables with symmetry properties given by $\mathrm{B}_{1 g}$ are compatible with the symmetry of the distorted phase, and therefore can be non-zero in this phase. They will be secondary spontaneous variables.

Figure 1 indicates that $x^{2}-y^{2}$ and $(x z, y z)$ transform according to $\mathrm{B}_{1 g}$ and $\mathrm{E}_{g}$, respectively. This means that the shear strain components $\left(\epsilon_{x z}, \epsilon_{y z}\right)$ have the symmetry properties of the active irrep, and could in principle be identified with the order parameter of the transition, while the strain

\footnotetext{
1 The computer-adapted irrep labels used by COPL and ISOTROPY differ from those used in some programs of the Bilbao Crystallographic Server. They coincide however with those employed by the programs SYMMODES and AMPLIMODES of the Bilbao server, as both of them use an adapted version of COPL.
} 
difference $\left(\epsilon_{x x}-\epsilon_{y y}\right)$ transforms according to $\mathrm{B}_{1 g}$. Being zero in the parent phase, $\left(\epsilon_{x x}-\epsilon_{y y}\right)$ becomes spontaneous, as a secondary variable, in the $C 2 / m$ phase. We have then a proper or pseudoproper ferroelastic transition (i.e. some strain component(s) has(have) the symmetry of the order parameter), and we expect the softness at the transition of the stiffness coefficients corresponding to $\epsilon_{x z}$ and $\epsilon_{y z}$, i.e. the elastic constants $C_{55}$ and $C_{66}$. The shear strain $\epsilon_{y z}$ (direction $(0,1)$ in $\mathrm{E}_{g}$ ) should become spontaneous in the $C 2 / m$ phase, deviating the monoclinic angle from $90^{\circ}$, as a primary order parameter effect. [Equivalently we could consider the domain related distortion with $\epsilon_{x z}$ (direction $(1,0)$ in $\left.\mathrm{E}_{g}\right)$ ]. On the other hand, the quantity $\left(\epsilon_{x x}-\epsilon_{y y}\right)$ is zero in the parent phase (cell parameters $a$ and $b$ are equal), while in the $C 2 / m$ phase $\left(\epsilon_{x x}-\epsilon_{y y}\right) \neq 0$ (cell parameter $a$ and $b$ become different), but as a secondary (weaker) effect.

As the index of $C 2 / m$ is 4 , four equivalent domain-related structures must exist. These domains should be distinguishable by the value of the order parameter (see eq. (2.3) above). From the structure having a spontaneous shear strain $\epsilon_{y z}=\epsilon^{\circ}$ and zero shear strain $\epsilon_{x z}:\left(0, \epsilon^{\circ}\right)$, we obtain the values of the spontaneous shear strains $\epsilon_{x z}$ and $\epsilon_{y z}$ in the other domains applying lost symmetry operations according to the matrices listed in Table $2:\left(2_{z}, 000\right) \rightarrow\left(0,-\epsilon^{\circ}\right),\left(4_{z}^{-}, 000\right) \rightarrow\left(\epsilon^{\circ}, 0\right),\left(4_{z}^{+}, 000\right) \rightarrow\left(-\epsilon^{\circ}, 0\right)$. Therefore, the primary spontaneous monoclinic strain can have opposite values in different domains, and can also have two different orientations with respect to the parent tetragonal phase, corresponding to the monoclinic axis being along $x$ or $y$, as listed in Table 3. On the other hand, the secondary spontaneous strain $\left(\epsilon_{x x}-\epsilon_{y y}\right)$ does not distinguish some of these domains, having the same value in domain pairs: $\left(2_{z}, 000\right) \rightarrow\left(\epsilon_{x x}-\epsilon_{y y}\right),\left(4_{z}^{-}, 000\right) \rightarrow-\left(\epsilon_{x x}-\epsilon_{y y}\right),\left(4_{z}^{+}, 000\right) \rightarrow-\left(\epsilon_{x x}-\epsilon_{y y}\right)$.

In general, the multistability and switching properties of a low-symmetry phase including the establishment and characterization of all possible domains can be determined if the symmetry of both the parent high-symmetry phase and the one of the distorted phase are known. The point groups of the two space groups, the so-called ferroic species, define the ferroic properties of the low-symmetry phase.

\subsection{The program SYMMODES}

The program SYMMODES [18] is designed to provide a basis of symmetry-adapted displacive modes for describing any displacive distortion relating a group-subgroup-related pair of phases. These modes, which are allowed to condense in the distorted phase can be primary (with their symmetry given by the active irrep) and secondary (with their symmetry given by secondary irreps having isotropy subgroups containing the observed space group). The program has been developed in collaboration with H. T. Stokes (Brigham Young University), and uses internally an adapted version of the program COPL mentioned above.

For each allowed irrep (either active/primary or secondary), and restricted to the required irrep subspace, SYMMODES lists a complete set of symmetry-adapted displacive modes for the Wyckoff orbit types chosen by the user. The program only requires as initial input the space group types of the high and low symmetry phase, $\mathcal{G}$ and $\mathcal{H}$, and their index. The program then determines all the different classes of subgroups $\mathcal{H}$ of $\mathcal{G}$, and the user has to choose the one relevant for the problem. A direct link to SUBGROUPGRAPH to obtain the graph of minimal subgroups is also available.

Once the relevant class of subgroups $\mathcal{H}$ is decided, the user chooses the set of Wyckoff positions, for which the basis of symmetry-adapted modes is desired. Note that the choice of unit cell for the $\mathcal{H}$-group done by the program may not coincide with the one wished, but an equivalent one should appear on the list. The program also allows to introduce a specific desired matrix transformation. For our $I 4 / \mathrm{mmm}$ example, we use this option to introduce the specific matrix transformation we have been considering above for $C 2 / \mathrm{m}$, and we choose the Wyckoff position $8 i$ to see the displacive modes that will be triggered by the transition for atoms on sites of this type. The resulting output is shown in Figure 6. It gives first a 'Symmetry Modes Summary' listing the irreps corresponding to the displacive modes allowed for the chosen Wyckoff position, and highlighting in bold letters the active irrep, i.e. the one having $C 2 / m$ as isotropy subgroup. We can see that modes of symmetry GM1+, GM2+ and GM5+ 


\section{Symmetry Modes for group - subgroup pair $14 / m m m(139)>C 2 / m(12)$}

Initial Data:

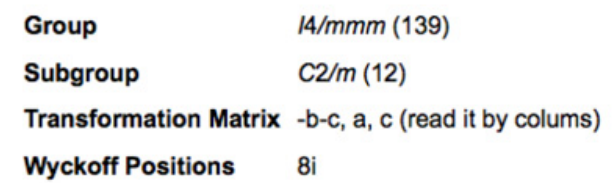

Symmetry Modes Summary

\begin{tabular}{|l|c|}
\hline WP & Modes \\
\hline $8 \mathrm{i}$ & $\mathrm{GM} 1+(1) \mathrm{GM} 2+(1) \mathrm{GM} 5+(1)$ \\
\hline Note: The primary mode is written in bold letters
\end{tabular}

$K$ vector: $\mathrm{GM}=(0,0,0)$

Irrep GM1+

Order Parameter (a)

Isotropy Subgroup H 139 /4/mmm D4h-17

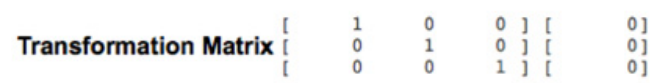

\begin{tabular}{|c|c|}
\hline WP 8i & Displacements \\
\hline$(x, 0,0)$ & $(1,0,0)$ \\
\hline$(-x, 0,0)$ & $(-1,0,0)$ \\
\hline$(0,-x, 0)$ & $(0,-1,0)$ \\
\hline$(0, x, 0)$ & $(0,1,0)$ \\
\hline
\end{tabular}

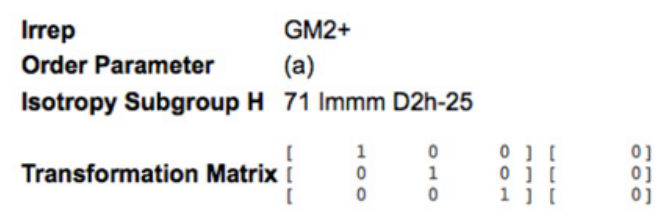

\begin{tabular}{|c|c|}
\hline WP 8i & Displacements \\
\hline \hline$(x, 0,0)$ & $(1,0,0)$ \\
\hline$(-x, 0,0)$ & $(-1,0,0)$ \\
\hline$(0,-x, 0)$ & $(0,1,0)$ \\
\hline$(0, x, 0)$ & $(0,-1,0)$ \\
\hline
\end{tabular}

Irrep GM5+

Order Parameter $\quad(a, 0)$

Isotropy Subgroup H $12 \mathrm{C} 2 / \mathrm{m} \mathrm{C} 2 \mathrm{~h}-3$

Transformation Matrix [ $\begin{array}{rrrrrrr}{[} & 0 & 1 & 0 & ] & {[} & 0] \\ {[} & -1 & 0 & 0 & ] & {[} & 0] \\ {[} & -1 & 0 & 1 & ] & {[} & 0]\end{array}$

\begin{tabular}{|c|c|}
\hline WP 8i & Displacements \\
\hline \hline$(x, 0,0)$ & $(0,0,0)$ \\
\hline$(-x, 0,0)$ & $(0,0,0)$ \\
\hline$(0,-x, 0)$ & $(0,0,1)$ \\
\hline$(0, x, 0)$ & $(0,0,-1)$ \\
\hline
\end{tabular}

Figure 6. Output of SYMMODES for the pair $I 4 / \mathrm{mmm} \longrightarrow C 2 / m(-\mathbf{b}-\mathbf{c}, \mathbf{a}, \mathbf{c} ; 000)$ and the Wyckoff positions $8 i$.

are allowed for $8 i$ sites in the distorted structures, in agreement with Table 6. The table also indicates (in parenthesis) the number of symmetry-modes that exists for each irrep. The output then lists for each irrep the corresponding isotropy subgroup with its transformation matrix, the eventual restriction on the direction within the irrep space (this is the data headed as 'order parameter'), and a set of linearly independent modes having this irrep as symmetry.

The form of the modes in Figure 6 is given as a column of triads indicating the correlated displacements of the atoms of the Wyckoff orbit extended to the primitive unit cell of the distorted phase. As the distorted phase in our example has the same primitive unit cell $\left(i_{k}=1\right)$ as the parent phase, the set of listed atomic positions is limited to 4 . The other atoms in the structure belonging to the same orbit are related by lattice translations to those listed in the output and their mode displacements will have the same displacements. The components of the atomic displacements describing the modes are given in the setting of the parent structure. Modes are not normalized and, in the case of existing several modes for the same irrep, they are not necessarily orthogonalized.

We can see in Figure 6 that for $8 i$ atoms, there is only a single mode with the symmetry of the active irrep. It involves displacements along the $z$-axis of only 2 of the 4 atoms within a primitive unit cell. Only atoms with non-zero $y$-component have non-zero displacements. Atoms with opposite $y$-components have opposite displacements along $z$. Modes GM1+ and GM2+ are restricted to the $x y$ plane. As it should be, the GM1+ mode displaces the atoms in such a way that it maintains the $I 4 / \mathrm{mmm}$ symmetry relation described in the first column. In contrast, the mode GM2+ mode breaks this relationship: $(x, 0,0)$ and $(0, x, 0)$ sites have $(0,1,0)$ and $(0,-1,0)$ displacements respectively. 


\begin{tabular}{|c|c|c|c|c|}
\hline \multicolumn{3}{|c|}{ Representative } & \multicolumn{2}{|c|}{ Subgroup Wyckoff position } \\
\hline No & group basis & subgroup basis & name[n] & representative \\
\hline 1 & $(x, 0,0)$ & $(0, x, 0)$ & \multirow{4}{*}{$4 g_{1}$} & $\left(0, y_{1}, 0\right)$ \\
\hline 2 & $(-x, 0,0)$ & $(0,-x, 0)$ & & $\left(0,-y_{1}, 0\right)$ \\
\hline 3 & $(x+1 / 2,-1 / 2,-1 / 2)$ & $(1 / 2, x+1 / 2,0)$ & & $\left(1 / 2, y_{1}+1 / 2,0\right)$ \\
\hline 4 & $(-x+1 / 2,-1 / 2,-1 / 2)$ & $(1 / 2,-x+1 / 2,0)$ & & $\left(1 / 2,-y_{1}+1 / 2,0\right)$ \\
\hline 5 & $(0, x, 0)$ & $(-x, 0,-x)$ & \multirow{4}{*}{$4 i_{1}$} & $\left(x_{2}, 0, z_{2}\right)$ \\
\hline 6 & $(0,-x, 0)$ & $(x, 0, x)$ & & $\left(-x_{2}, 0,-z_{2}\right)$ \\
\hline 7 & $(1 / 2, x-1 / 2,-1 / 2)$ & $(-x+1 / 2,1 / 2,-x)$ & & $\left(x_{2}+1 / 2,1 / 2, z_{2}\right)$ \\
\hline 8 & $(1 / 2,-x-1 / 2,-1 / 2)$ & $(x+1 / 2,1 / 2, x)$ & & $\left(-x_{2}+1 / 2,1 / 2,-z_{2}\right)$ \\
\hline
\end{tabular}

Figure 7. Detailed splitting of orbits $8 i$ for the symmetry break $I 4 / m m m \longrightarrow C 2 / m(-\mathbf{b}-\mathbf{c}, \mathbf{a}, \mathbf{c} ; 000)$ as obtained by SYMMODES.

From Figure 6 one expects that the atoms $8 i$ of type $(0, x, 0)$ in the $I 4 / \mathrm{mmm}$ structure and their related ones by the $I$ centring will suffer displacements along the $c$ axis much stronger than along the $y$ axis, the only two directions allowed (always working in the tetragonal setting), because the $z$ displacements correspond to the primary mode with the symmetry of the active irrep, and will be in principle related with the transition mechanism. The atoms with positions $(x, 0,0)$ and the related ones by the $I$ centring, on the other hand, will only have secondary displacements along the $x$-direction, that maintain the symmetry relation among them.

SYMMODES also allows examining the splitting of the Wyckoff orbit due to the symmetry decrease, by means of a link to the program WYCKSPLIT [3, 19] of the server. The table shown in Figure 7 shows the corresponding output for an $8 i$ orbit. The last column in Figure 7 describes the generic positions of the two splitted orbits in the subgroup basis, while the third column indicates the corresponding position in the higher $I 4 / \mathrm{mmm}$ structure in the same basis. One can see that the first four positions forming the $4 g_{1}$ orbit do not really break the tetragonal symmetry; their only allowed variation of the $y$ component (in the subgroup basis) is also compatible with the tetragonal space group. On the other hand, the other four positions forming the $4 i_{1}$ orbit introduce an additional degree of freedom by breaking the correlation between the $x$ and $z$ components (in the subgroup basis). The number of symmetry free components in the two orbits is 3 . This should coincide with the number of distinct modes listed in Figure 6 . The number of modes is equal to the number of degrees of freedom for the $8 i$ atomic positions in the subgroup, and this is the number of free parameters in the resulting Wyckoff orbits in the subgroup symmetry.

Even if we are not interested in the displacive modes present in the distorted phase, SYMMODES can also be used to obtain important information on the active and secondary irreps relevant for a given symmetry break. The information is more complete than in COPL as the transformation matrices for each isotropy subgroup are also listed. But a caution note should be added: SYMMODES only lists the compatible irreps which can be associated to displacive modes of the Wyckoff positions chosen. If we are interested in all the irreps compatible with the symmetry reduction, it is convenient to choose a general Wyckoff orbit, in order to have as many symmetry allowed irreps as possible. In most cases this will be sufficient to get all compatible irreps, but in some cases some compatible irreps may not be involved in any atomic displacements, and they will not be listed by SYMMODES. 
SYMMODES can for instance be used to see that from the six space groups listed in Table 5, only the groups Pnma and Pmma are isotropy subgroups of $F m \overline{3} m$ and a single active irrep can be identified (this has been indicated in the fourth column of the table).

\section{SYMMETRY-MODE DECOMPOSITION OF DISTORTED STRUCTURES. THE PROGRAM AMPLIMODES}

The natural language to deal with the static frozen distortions present in displacively distorted structures is the one of modes. Modes are collective correlated atomic displacements fulfilling certain symmetry properties. Structural distortions in distorted structures can be decomposed into contributions of modes with different symmetries given by irreps of the parent space group. One can then distinguish primary and secondary (induced) distortions, which will have in general quite different weights in the structure, and will respond differently to external perturbations. In general, the use of symmetry-adapted modes in the description of distorted structures introduces a natural physical hierarchy among the structural parameters. This can be useful not only for investigating the physical mechanisms that stabilize these phases, but also for pure crystallographic purposes. The set of structural parameters used in a mode description of a distorted phase will in general be better adapted for a controlled refinement of the structure, or for instance for comparative studies between different materials or for ab-initio calculations. For a review of the applications of the symmetry-mode approach in structural problems see [20]. AMPLIMODES [5] is a computer program available on the Bilbao Crystallographic Server that can perform the symmetry-mode analysis of any distorted structure of displacive type. The analysis consists in decomposing the symmetry-breaking distortion present in the distorted structure into contributions from different symmetry-adapted modes. Given the high- and the low-symmetry structures, AMPLIMODES determines the atomic displacements that relate them, defines a basis of symmetry-adapted modes, and calculates the amplitudes and polarization vectors of the distortion modes of different symmetry (different irrep) frozen in the structure. The program uses a mode parameterization that is as close as possible to the crystallographic conventions, using an asymmetric unit of the low-symmetry structure for describing modes and distortions. AMPLIMODES uses internally SYMMODES to produce the basis of symmetry-adapted modes to be used in the decomposition of the structural distortion, but instead of describing the modes in the high-symmetry setting, it works in the setting of the subgroup. A detailed account of the mathematical formalism and parameterization of the program can be found in [5].

In order to see the possibilities of the program the simplest approach is to see in detail an example. Let us consider the structure of $\mathrm{SrMnO}_{3}$ at $100 \mathrm{~K}$ that has been recently reported in [21]. Above $350 \mathrm{~K}$ this compound is known to have hexagonal symmetry with space group $P 6_{3} / m m c$, while below this temperature a slight distortion happens and the structure is orthorhombic, with space group $C 222_{1}$, i.e. a subgroup of $\mathrm{P6}_{3} / \mathrm{mmc}$ related by the transformation $(\mathbf{a}, \mathbf{a}+2 \mathbf{b}, \mathbf{c} ; 000)$. The structures of these two phases were determined from powder diffraction data (combining both neutron and synchrotron data). The orthorhombic phase is metrically hexagonal with no appreciable orthorhombic strain, and a powder diffraction refinement in these circumstances is bound to be especially problematic. Thus, the refinement of the orthorhombic phase was done introducing some constraints that reduced the number of free positional atomic parameters. The number of linear constraints explicitly mentioned in the article were five, which in principle would reduce the number of positional degrees of freedom from 13 to 8 .

The reason for the specific constraints that were used in the structural model were not explained in the article. An analysis of the structure with AMPLIMODES makes clear the physical basis of this constrained refinement but also shows some shortcomings of the specific constraints that were used. The application of the program only requires as input the structures of the two phases and the basis transformation relating them. This is sufficient to produce the symmetry-mode decomposition of the $C 222_{1}$ structure, which is summarized in Table 7. One can see that the 13-dimensional configuration space of the $C 222_{1}$ distortion can be divided in 4 subspaces corresponding to four distinct irreps at the Brillouin zone centre. The active irrep is GM5- (see Figure 8), which spans a 6-dimensional 


\section{EPJ Web of Conferences}

Table 7. Summary of the symmetry-mode decomposition of the $C 222_{1}$ structure of $\mathrm{SrMnO}_{3}$ reported in [21] with respect to the $P 6_{3} / \mathrm{mmc}$ structure at $350 \mathrm{~K}$. The table indicates the amplitudes in $\AA$ of the different irrep distortions allowed by symmetry, indicating for each irrep, its wave vector, label, direction within the irrep space, isotropy subgroup and dimension of the distortion subspace.

\begin{tabular}{cccccc}
\hline & & \multicolumn{3}{c}{ Isotropy } \\
k-vector & Irrep & Direction & Subgroup & Dimension & Amplitude $(\AA)$ \\
\hline$(0,0,0)$ & GM1+ & $(\mathrm{a})$ & $P 6_{3} / m m c$ & 2 & 0.04 \\
$(0,0,0)$ & GM5+ & $(-0.500 \mathrm{a},-0866 \mathrm{a})$ & $C m c m$ & 4 & 0.00 \\
$(0,0,0)$ & GM1 & $(\mathrm{a})$ & $P 6_{3} 22$ & 1 & 0.00 \\
$(0,0,0)$ & GM5- & $(\mathrm{a}, 0)$ & $C 222_{1}$ & 6 & 0.39 \\
\hline
\end{tabular}

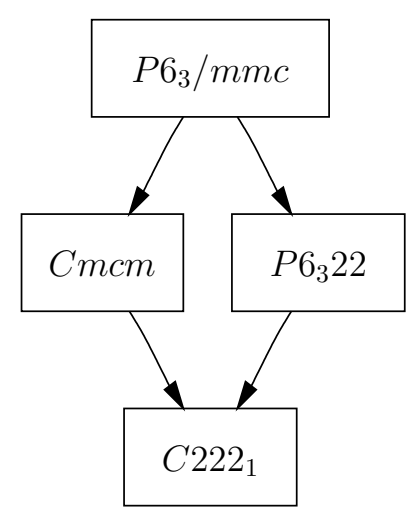

Figure 8. Graph of maximal subgroups connecting the parent space group $P 6_{3} / m m c$ and the symmetry $C 222_{1}$ of $\mathrm{SrMnO}_{3}$.

subspace within the $C 222_{1}$ configuration space. From the amplitudes of the different symmetry-adapted distortions one can see that the refined structure is described by only a distortion corresponding to the active irrep GM5- (plus a very weak GM1 + distortion, compatible with the parent $P 6_{3} / m m c$ symmetry). The secondary distortions of symmetry GM5+ and GM1-, which correspond to five additional degrees of freedom compatible with supergroups of $C 222_{1}$ (see Figure 8), have zero amplitudes. The five linear constraints used in the refinement of the atomic positions seem therefore to correspond to the fixing of these two secondary distortions to zero. We expect indeed in a groupsubgroup phase transition that the primary distortion corresponding to the active irrep is dominant. The condensation of the other irrep distortions, being a secondary effect, have often amplitudes one order of magnitude smaller. If a decrease of the number of refinable parameters is critical for a successful refinement, it is then a clever approach to set to zero these secondary irrep distortions. This has certainly been done in this structure refinement, but if we look in detail to the output of AMPLIMODES, we will see that some of the contraints are only indirectly producing the fixing to zero of the secondary distortions and have been combined with an unjustified additional constraint of the primary distortion GM5-.

Table 8 shows the so-called 'reference structure' used by the program. It is the parent $P 6_{3} / m m c$ structure described in the lower space group $C 222_{1}$. The asymmetric unit defined in this Table is the one that the program uses to describe all the symmetry-modes. A set of displacements of the atoms within this asymmetric unit (expressed in relative units) is sufficient to describe any symmetry-mode compatible with the $C 222_{1}$ symmetry. In general, AMPLIMODES gives the symmetry-modes normalized to $1 \AA$ within a primitive unit cell of the reference structure (the so-called polarization vector of the mode), plus a global amplitude in $\AA$. 
Contribution of Symmetries in Condensed Matter

Table 8. Reference structure for the irrep distortions in the $C 222_{1}$ phase of $\mathrm{SrMnO}_{3}$.

\begin{tabular}{|c|c|c|c|c|}
\hline \multicolumn{5}{|c|}{ Space group $C 222_{1} a=5.461 \AA b=9.4587 \AA c=9.093 \AA$} \\
\hline \multirow{2}{*}{$\begin{array}{l}\text { Atom } \\
\text { Sr1 }\end{array}$} & \multirow{2}{*}{$\begin{array}{c}\begin{array}{c}\text { Wyckoff } \\
\text { positions }\end{array} \\
4 \mathrm{a}\end{array}$} & \multicolumn{3}{|c|}{ Coordinates } \\
\hline & & 0.000000 & 0.000000 & 0.000000 \\
\hline $\mathrm{Sr} 2$ & $4 b$ & 0.000000 & 0.333333 & 0.250000 \\
\hline Mn1 & $8 c$ & 0.000000 & 0.333333 & 0.612640 \\
\hline $\mathrm{O} 1$ & $4 a$ & 0.500000 & 0.000000 & 0.000000 \\
\hline O1_2 & $8 c$ & 0.750000 & 0.250000 & 0.000000 \\
\hline $\mathrm{O} 2$ & $8 c$ & 0.772130 & 0.409290 & 0.750000 \\
\hline O2_2 & $4 b$ & 0.000000 & 0.181420 & 0.750000 \\
\hline
\end{tabular}

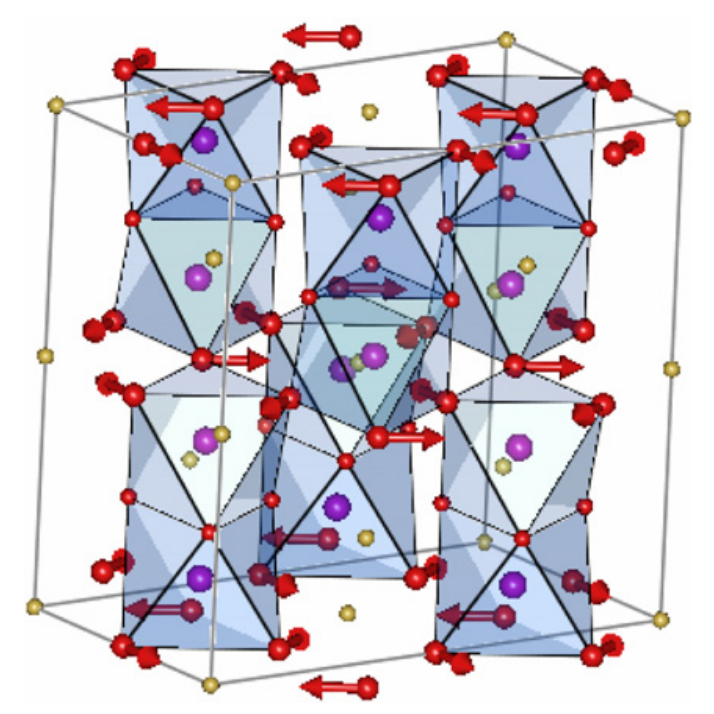

Figure 9. GM1-symmetry mode allowed in the $C 222_{1}$ phase of $\mathrm{SrMnO}_{3}$ (figure done with FullProf Studio [23]).

Figure 9 depicts the only existing mode of GM1- symmetry, which in principle could be present in the $C 222_{1}$ distortion. It corresponds to antiphase rotations around the $c$ axis of the oxygen triangles formed by $\mathrm{O} 1$ and $\mathrm{O} 1 \_2$ atoms, on the columns of oxygen octahedra. This mode involves specific correlated displacements of the $\mathrm{O} 1$ and $\mathrm{O} 1 \_2$ atoms along the $x$ and along the $x$ and $y$ directions of the orthorhombic setting, respectively, which decrease the symmetry to $P 6_{3} 22$, a supergroup of $C 222_{1}$. However, the only explicit constraint introduced for these atoms in [21] was to fix to zero the $y$ displacement of the O1_2 atom. This is clearly not equivalent to setting to zero the GM1distortion, since not only GM1- can contribute to the y displacement of the O1_2 atom, but also the GM5- distortion. Two of the basis modes of symmetry GM5- used by AMPLIMODES (modes 3 and 5 in Table 9) include $y$-displacements of the O1_2 atom. Therefore the cancelling of the GM1distortion in the refined structure must have been achieved by introducing some additional restriction in the possible form of the GM5- distortion. This becomes evident inspecting the GM5- distortion derived by AMPLIMODES. The GM5- distortion, which multiplied by the amplitude of 0.39 and added to the reference structure given in Table 8 essentially describes the reported $C 222_{1}$ structure, is given in crystallographic form in Table 10, and is shown schematically in Figure 10. The mode has in principle six degrees of freedom corresponding to the six orthonormal basis modes listed in Table 9. However, the 
Table 9. The six basis modes corresponding to the irrep GM5 - used in AMPLIMODES to describe the GM5distortion present in the $C 222_{1}$ structure of $\mathrm{SrMnO}_{3}$. Displacements are normalized to $1 \AA$ within a $C 222_{1}$ primitive unit cell, and are described in relative units with respect to the conventional $C 222_{1}$ unit cell of the reference structure.

\begin{tabular}{llrrr}
\hline & Atom & \multicolumn{1}{c}{$\delta x$} & \multicolumn{1}{c}{$\delta y$} & \multicolumn{1}{c}{$\delta z$} \\
\hline Mode 1 (Sr1) & Sr1 & 0.129483 & 0.000000 & 0.000000 \\
Mode 2 (Mn1) & Mn1 & 0.091558 & 0.000000 & 0.000000 \\
Mode 3 (O1) & O1 & 0.105722 & 0.000000 & 0.000000 \\
& O1_2 & 0.026431 & -0.026431 & 0.000000 \\
Mode 4 (O1) & O1 & 0.000000 & 0.000000 & 0.000000 \\
& O1_2 & 0.000000 & 0.000000 & 0.054987 \\
Mode 5 (O1) & O1 & 0.000000 & 0.000000 & 0.000000 \\
& O1_2 & -0.079292 & -0.026431 & 0.000000 \\
Mode 6 (O1) & O1 & 0.000000 & 0.000000 & -0.054987 \\
& O1_2 & 0.000000 & 0.000000 & 0.000000 \\
\hline
\end{tabular}

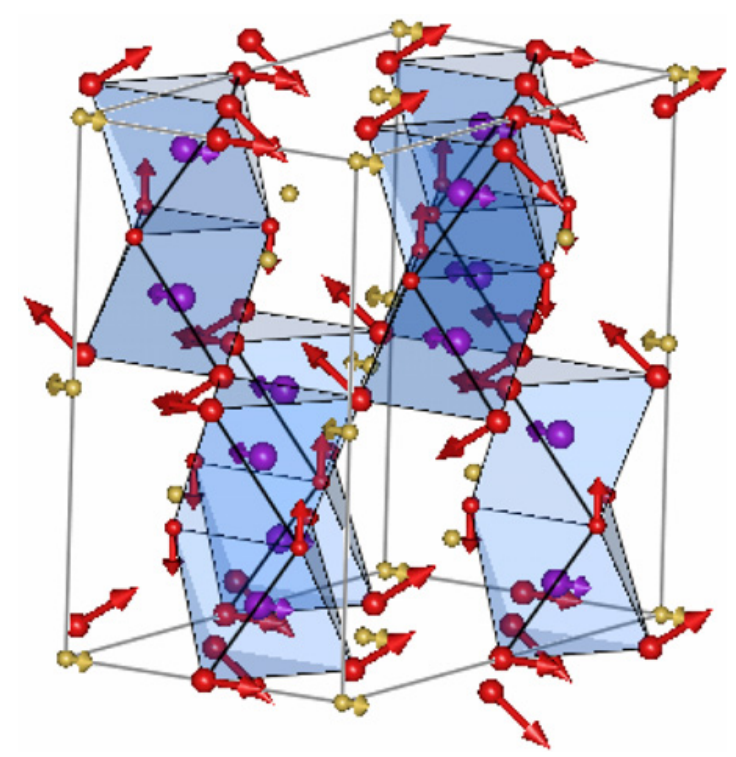

Figure 10. Polarization vector in an arbitrary scale of the GM5- distortion that describes the refined $C 222_{1}$ structure of $\mathrm{SrMnO}_{3}$ (figure done with FullProf Studio [23]).

mode described in Table 10 has only five distinct parameters, since the $x$-displacement of the atoms O1 and O1_2 are equal. This equality is not forced by the GM5 - symmetry. In fact, one can see in Table 9 that also $y$-displacements of the atom O1_2 would be symmetry allowed. Hence the GM5- distortion was somehow constrained in the refinement of this structure. The decomposition of the distortion into the six basis modes, also indicated in Table 10, shows that the modes 3 and 5 of Table 9 have exactly the same amplitude, making the O1_2 y-displacement zero. This also forces the equality of the $x$-displacements of the $\mathrm{O} 1$ and $\mathrm{O} 1 \_2$.

The refinement of the $C 222_{1}$ structure of $\mathrm{SrMnO}_{3}$ seems therefore to have been performed with an additional implicit constraint on the primary distortion. By means of simple clever fixing of some 


\section{Contribution of Symmetries in Condensed Matter}

Table 10. Normalized polarization vector of the GM5- distortion present in the $C 222_{1}$ structure of $\mathrm{SrMnO}_{3}$. Displacements are in relative units (normalization to $1 \AA$ of all displacements within a reference primitive unit cell). This corresponds to a 6-dimensional vector with components $(0.1893,-0.3429,0.5119,0.3946,-0.5119$, $-0.4085)$ in the basis of the 6 modes indicated in Table 9. Note the exact opposite values of the components corresponding to modes 3 and 5, which make null the $y$ displacement of O1_2.

\begin{tabular}{lrcc}
\hline atom & \multicolumn{1}{c}{$\delta x$} & $\delta y$ & $\delta z$ \\
\hline Sr1 & 0.0245 & 0.0000 & 0.0000 \\
Sr2 & 0.0000 & 0.0000 & 0.0000 \\
Mn1 & -0.0314 & 0.0000 & 0.0000 \\
O1 & 0.0541 & 0.0000 & 0.0000 \\
O1_2 & 0.0541 & 0.0000 & 0.0217 \\
O2 & 0.0000 & 0.0000 & 0.0225 \\
O2_2 & 0.0000 & 0.0000 & 0.0000 \\
\hline
\end{tabular}

of the atomic coordinates or forcing simple relations among them, the secondary distortions summing five degrees of freedom could be set to zero, but this method also indirectly introduced a constraint of the primary distortion, which has no apparent justification. The linear constraint equivalent to fixing to zero the GM1- mode, without constraining the form of the GM5- distortion would have required to introduce a linear relation between three atomic coordinates, namely the $x, y$ coordinates of $01 \_2$ and the $x$ coordinate of $\mathrm{O} 1$.

The controlled switching off (or on) of distortions associated to specific irreps, in particular those corresponding to secondary distortions, in the refinement of distorted structures, can now be done automatically using FullProf [22] combined with AMPLIMODES. In collaboration with J. RodriguezCarvajal we have included in AMPLIMODES an option, which creates a special output file to be directly used by FullProf. By this means, FullProf can now use in the refinement process the amplitudes of the basis of symmetry modes defined by AMPLIMODES as refinable positional parameters, alternative to the atomic coordinates. In general, the expected hierarchy among the distortions of different symmetry and the essential invariance of their polarization vectors can make very advantageous a direct refinement of the amplitudes of a basis of symmetry modes, as collective coordinates, instead of the usual individual atomic coordinates. The significance of the weak distortions associated with secondary modes can be monitored and if necessary fixed to zero. The combined use of AMPLIMODES and FullProf can also be used to visualize and generate figures of the distortion modes, and of the basis of symmetry-adapted modes.

\section{STRUCTURAL PHASE TRANSITIONS WITH SEVERAL ACTIVE IRREPS}

There are some symmetry breaks that do not fulfill the Landau postulate of having a single active irrep. In other words, the symmetry reduction cannot be explained by a single irrep, as the symmetry of the distorted phase is not an isotropy subgroup of the parent space group. If the transition is discontinuous or first order, there are mechanisms that can explain the breaking of the Landau postulate and predict the simultaneous condensation of two irreps. However, these situations are rare, and some plausible explanation for such cases that one should check carefully, is the possibility that the condensation of the two active irreps is in fact stepwise and an intermediate phase has been overlooked.

Independently of the existence or not of an intermediate phase, the existence of two active irreps implies a scenario, where several degrees of freedom of the structure are unstable in the high-symmetry configuration. This is bound to produce rich phase diagrams. Let us consider the example of the Aurivillius compound $\mathrm{SrBi}_{2} \mathrm{Ta}_{2} \mathrm{O}_{9}$ [24]. The compound is known to have a tetragonal $I 4 / \mathrm{mmm}$ phase at high-temperatures with $\mathrm{Z}=2$, and at room temperature crystallizes in the subgroup $C m c 2_{1}\left(\mathbf{c}, \mathbf{a}-\mathbf{b}, \mathbf{a}+\mathbf{b} ; \frac{1}{4}, \frac{1}{4}, 0\right)$ with $\mathrm{Z}=4$. Using SYMMODES we can obtain the graph (see Figure 11) 


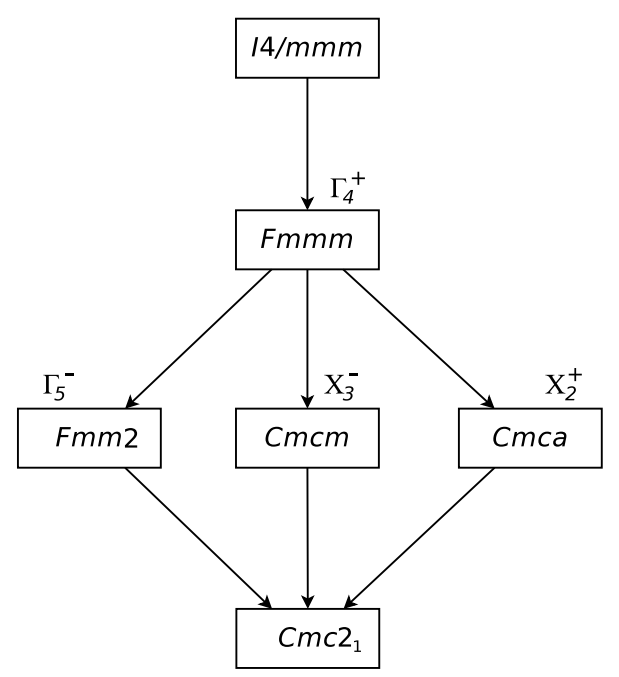

Figure 11. Graph of maximal subgroups connecting the space group $I 4 / \mathrm{mmm}$ and its subgroup $\mathrm{Cmc} 2_{1}$ $\left(\mathbf{c}, \mathbf{a}-\mathbf{b}, \mathbf{a}+\mathbf{b} ; \frac{1}{4}, \frac{1}{4}, 0\right)$ (or equivalent). Note that the group $C m c a$ (N. 64) is labelled $C m c e$ in the new convention of the 5th edition of the International Tables for Crystallography, Vol. A [25].

of the intermediate subgroups relating this group-subgroup pair (note that there are two $C m c 2_{1}$ classes with the same type of cell transformation, and only distinguishable by the origin shift. So one has to take care of choosing the correct one).

For each of the subgroups, we have indicated in Figure 11 the corresponding active irrep, if existing. The graph shows then that all subgroups are isotropy subgroups for some irrep, except for the observed symmetry. The room temperature phase requires therefore several active irreps. How many? Just two. The effective symmetry resulting from the presence of two of the distortions is the intersection of the two corresponding isotropy subgroups, and this is given in the graph by their first common subgroup. Hence, it is sufficient to consider any pair of the distortions $\Gamma_{5}^{-}, \mathrm{X}_{3}^{-}$and $\mathrm{X}_{2}^{+}$, to reach the symmetry $C m c 2_{1}$. Only the irrep $\Gamma_{4}^{+}$cannot be relevant for producing the observed symmetry $C m c 2_{1}$, i.e. its addition to any of the other ones, does not decrease further the symmetry.

We have therefore three possible irreps among which two must be the active ones. Symmetry considerations cannot go further. Only a quantitative analysis of the structure, ab-initio calculations, or the thermal behavior of the system can indicate which of the three irreps are really the active irreps in this specific case. The distortions having the symmetry of the active irrep are expected to have larger amplitudes, and be the ones that are more unstable in the high-symmetry configuration. For instance, a decomposition of the $C m c 2_{1}$ structure of $\mathrm{SrBi}_{2} \mathrm{Ta}_{2} \mathrm{O}_{9}$ in terms of symmetry-modes, done with AMPLIMODES (see Section 5), shows that the distortions of symmetry $\Gamma_{5}^{-}$and $\mathrm{X}_{3}^{-}$have much larger amplitude than the one for the irrep $\mathrm{X}_{2}^{+}$. Furthermore, the $\mathrm{X}_{3}^{-}$distortion is significantly larger than the $\Gamma_{5}^{-}$. Therefore we can identify $\mathrm{X}_{3}^{-}$and $\Gamma_{5}^{-}$as the active irreps. The additional $\mathrm{X}_{2}^{+}$spontaneous distortion present in the experimental structure can be considered a secondary effect, basically induced by the presence of the other two primary distortion modes [24].

As the $X_{3}^{-}$distortion is the largest one we expect it to be the one that thermalizes to zero value at higher temperature, and therefore we can infer that an intermediate phase with symmetry $\mathrm{Cmcm}$ is highly probable, corresponding to the presence of only this active irrep. Indeed this intermediate phase has been observed. The symmetry of the other primary distortion $\Gamma_{5}^{-}$corresponds to the irrep label $\mathrm{E}_{u}$ in the notation of POINT (see Figure 1). It corresponds to the symmetry properties of a vector on the plane $x y$, and therefore is a polar instability, that can produce a spontaneous polarization (its isotropy subgroup $F m m 2$ is polar along the $(1,1,0)$ direction in the tetragonal setting). This $\Gamma_{5}^{-}$polar distortion 


\section{Contribution of Symmetries in Condensed Matter}

is then the mechanism that causes the loss of the inversion centre and the ferroelectric properties of the room temperature phase.

\section{CONCLUSIONS}

We have briefly outlined some basic symmetry issues that underlie the analysis and understanding of the properties of solid-solid phase transitions having group-subgroup relations. In particular we have shown how representation analysis can be systematically applied, introducing a powerful framework for the study of both the physical and the structural properties of the distorted phases. We have shown by means of several examples, how this symmetry analysis can be done using computer tools freely available in internet. The use of these tools is straightforward and does not require a deep knowledge of group theory.

In many ways, the investigation of structural phase transitions by means of representation analysis is analogous to the representation analysis commonly employed in the study of magnetic transitions and magnetic structures, and has similar advantages. However, in the case of structural phase transitions, the application of group representation theory has somewhat a higher degree of complexity as it has to be combined necessarily with ordinary space group symmetry arguments. This has probably hampered a generalization of its use. Hopefully the newly available computer tools discussed here will help to change this situation.

This work has been supported by the Spanish Ministry of Science and Innovation (project MAT2008-05839) and the Basque Government (project IT-282-07).

\section{References}

[1] L. D. Landau \& E. M. Lifshitz. Statistical Physics. (Oxford: Pergamon Press, 1969).

[2] Y. Izyumov \& V. Syromyatnikov, V. (1990). Phase transitions and crystal symmetry. (Dordrecht: Kluwer Academic Publishers, 1990).

[3] M. I. Aroyo, J. M. Perez-Mato, C. Capillas, E. Kroumova, S. Ivantchev, G. Madariaga, A. Kirov \& H. Wondratschek, Z. Kristallogr. 221, 15-27 (2006).

[4] M. I. Aroyo, A. Kirov, C. Capillas, J. M. Perez-Mato \& H. Wondratschek, Acta Cryst. A62, 115128 (2006).

[5] D. Orobengoa, C. Capillas, M. I. Aroyo \& J. M. Perez-Mato, J. Appl. Cryst., 42, 820-833 (2009).

[6] J. C. Toledano \& P. Toledano. The Landau Theory of Phase Transitions. (Singapore: World Scientific, 1987).

[7] H. T. Stokes \& D. M. Hatch. Isotropy subgroups of the 230 crystallographic space groups. (Singapore: World Scientific, 1988).

[8] V. Janovec \& J. Privratska, J. (2003). 3.4. Domain Structures in International Tables for Crystallography, vol. D (Dordrecht: Kluwer Academic Publishers, 2003).

[9] C. J. Howard \& H. T. Stokes. Acta Cryst. B 54, 782-789 (1998).

[10] P. Tomaszewski. Phase Transitions, 38(3, Part B), 127-220 (1992).

[11] H. T. Stokes \& D. M. Hatch. (2002). ISOTROPY Department of Physics and Astronomy Bringham Young University, Provo, USA. (http://stokes.byu.edu/isotropy.html)

[12] B. J. Campbell, H. T. Stokes, D. E. Tanner \& D. M. Hatch. J. Appl. Cryst. 39607 (2006).

[13] S. Pekker et al. Nature Mat. 4, 764 (2005).

[14] N. M. Nernes et al. J. Phys. Chem. B 113, 2042 (2009)

[15] H. T. Stokes \& D. M. Hatch. J. Appl. Cryst., 35, 379 (2002).

[16] S. Ivantchev, E. Kroumova, G. Madariaga, J. M. Perez-Mato and M. I. Aroyo, J. Appl. Cryst. 33 1190 (2000). 
[17] A. B. Harris, arXiv: 0902.2388v1 [cond-mat.mtrl-sci] (http://arxiv.org/abs/0902.2388v1).

[18] C. Capillas, E. Kroumova, M. I. Aroyo, J. M. Perez-Mato, H. T. Stokes \& D. M. Hatch, J. Appl. Cryst., 36, 953-954 (2003).

[19] E. Kroumova, M. I. Aroyo, J. M. Perez-Mato, J. Appl. Cryst. 31646 (1998).

[20] J. M. Perez-Mato, D. Orobengoa, M. I. Aroyo, Acta Cryst. A 66, 558-590 (2010).

[21] A. Daoud-Aladine, C. Martin, L. C. Chapon, M. Hervieu, K. S. Knight, M. Brunelli \& P. G. Radaelli, Phys. Rev. B 75104417 (2007).

[22] J. Rodríguez-Carvajal, Phys. B Condens. Matter, 192, 55 (1993). The programs of the FullProf Suite and their corresponding documentation can be obtained from the Web at http://www.ill.eu/sites/fullprof/.

[23] L. C. Chapon \& J. Rodríguez-Carvajal, 2005 unpublished. FullProf Studio is a program of the FullProf Suite the most recent version is freely available in the site given in [22].

[24] J. M. Perez-Mato, M. I. Aroyo, A. García, P. Blaha, K. Schwarz, J. Schweifer \& K. Parlinski. Phys. Rev. B 70(21), 214111 (2004).

[25] International Tables of Crystallography, Vol. A Space-Group Symmetry. Edited by T. Hahn, 5th ed. (Dordrecht: Kluwer Academic Publishers, 2002). 